\title{
Destructive Arthritis
}

National Cancer Institute

\section{Source}

National Cancer Institute. Destructive Arthritis. NCI Thesaurus. Code C70634.

Arthritis associated with the destruction of the cartilage and the articular surface of the bone of the joint. This process severely interferes with movement in the joint. 